\title{
Masses of c-type RR Lyrae Variables in Globular Clusters
}

\author{
C. Cacciari ${ }^{1}$, A. Bruzzi ${ }^{2}$ \\ ${ }^{1}$ Bologna Observatory, Italy, ${ }^{2}$ University of Bologna, Italy,
}

The mass of RR Lyrae variables has been a controversial problem for about a decade: while the stellar evolution theory predicts masses ranging between 0.65 and $0.75 \mathrm{M}_{\odot}$ for Oosterhoff type I and II clusters respectively, the stellar pulsation theory predicts smaller masses ( 0.55 and 0.65 respectively) using the double-mode pulsators. Simon (1990, M.N.R.A.S. 246, 70), comparing hydrodynamical models with observed stars by means of Fourier parameters, has found relations between the stellar mass and its luminosity, pulsation period, Helium content and Fourier parameter $\phi_{31}$. Combining his equations we obtain:

$$
\log M=0.590 \log L-0.052 \phi_{31}-0.056 \log P-1.068
$$

from which one can estimate the stellar mass by using the observable quantities $P$ and $\phi_{31}$ and a luminosity scale, e.g. the one derived by Cacciari, Clementini and Fernley (1992, Astrophys. J. in press). We have selected the clusters with a good number of c-type variables and available photometry in the literature, except for M3 for which we have used our own CCD photometry (Carretta 1991, Thesis, University of Bologna): the list includes M3, M5, M62, NGC3201, NGC4147 and NGC6171 in the Oosterhoff I group, and M15, M53, M68 and NGC4833 in the Oosterhoff II group, for a total of 76 and 74 stars respectively. For each star the parameter $\phi_{31}$ was calculated and the stellar mass was derived. The average values of mass were $0.53 \pm 0.07 \mathrm{M}_{\odot}(\mathrm{OoI})$ and $0.58 \pm 0.09 \mathrm{M}_{\odot}(\mathrm{OoII})$, with $\mathrm{M} \sim-0.05[\mathrm{Fe} / \mathrm{H}]+0.47$ (typical error of $15 \%$ on the average mass per cluster). The main conclusions can be summarized as follows:

- These values of mass are a direct consequence of the adopted luminosity scale. They appear rather low, and in order to reconcile them with the larger values predicted by the evolution theory the zero-point of the luminosity scale should be brighter: an increase in mass by $\sim 10 \%$ would increase the luminosity by $\sim 0.2$ mag. This is in agreement with the suggestion made by several other authors in this conference. The difference in mass between OoI and II groups is smaller than predicted by the classical pulsation and evolution theories, and more in agreement with the results of calculations which make use of enhanced (metal) opacities.

- Simon's $\phi_{31}$ method, although potentially very interesting when applied on a statistically significant sample of stars, is not sufficiently accurate for deriving reliable masses of individual stars. 